\title{
Tangence
}

\section{Ubi libertas, ibi patria : les échanges interculturels dans les calendriers germano-américains aux XVIII ${ }^{\mathrm{e}}$ et $\mathrm{XIX}^{\mathrm{e}}$ siècles Ubi libertas, ibi patria: Intercultural Exchanges in German-American Calendars in the 18th and 19th Centuries}

\section{York-Gothart Mix}

Numéro 72, été 2003

Transferts culturels entre l'Europe et l'Amérique du Nord aux XVIII ${ }^{\mathrm{e}}$ et XIX ${ }^{\mathrm{e}}$ siècles. Circulation des savoirs, réappropriations formelles, réécritures

URI : https://id.erudit.org/iderudit/009091ar

DOI : https://doi.org/10.7202/009091ar

Aller au sommaire du numéro

Éditeur(s)

Tangence

ISSN

0226-9554 (imprimé)

1710-0305 (numérique)

Découvrir la revue

Citer cet article

Mix, Y.-G. (2003). Ubi libertas, ibi patria : les échanges interculturels dans les calendriers germano-américains aux XVIII ${ }^{\mathrm{e}}$ et XIX ${ }^{\mathrm{e}}$ siècles. Tangence, (72),

41-55. https://doi.org/10.7202/009091ar
Résumé de l'article

Les seuls travaux relativement récents qui proposent un point de vue d'ensemble sur les relations littéraires entre l'Amérique du Nord et l'Allemagne n'ont pas tenu compte d'une importante source textuelle pourtant inscrite au coeur du discours social le plus quotidien et étroitement liée aux questions d'identité et de différences culturelles : celle des calendriers populaires allemands publiés en Pennsylvanie à partir de 1731 et ailleurs en Amérique du Nord. Un tel corpus définit quatre objets qui seront de la plus haute importance pour toute recherche future. Il s'agit d'étudier d'abord les caractéristiques spécifiques du média et les différences génériques qui s'expriment dans le calendrier germano-américain ; deuxièmement, l'ensemble des modèles identitaires véhiculés par ces périodiques ; troisièmement, les formes possibles de communication interculturelle ; et, finalement, la relation entre adaptation culturelle et autonomie. 


\section{Ubi libertas, ibi patria: les échanges interculturels dans les calendriers germano-américains aux XVIII ${ }^{\mathrm{e}}$ et XIX ${ }^{\mathrm{e}}$ siècles ${ }^{1}$}

York-Gothart Mix, Université de Marburg

Les seuls travaux relativement récents qui proposent un point de vue d'ensemble sur les relations littéraires entre l'Amérique du Nord et l'Allemagne n'ont pas tenu compte d'une importante source textuelle pourtant inscrite au cœur du discours social le plus quotidien et étroitement liée aux questions d'identité et de différences culturelles: celle des calendriers populaires allemands publiés en Pennsylvanie à partir de 1731 et ailleurs en Amérique du Nord. Un tel corpus définit quatre objets qui seront de la plus haute importance pour toute recherche future. II s'agit d'étudier d'abord les caractéristiques spécifiques du média et les différences génériques qui s'expriment dans le calendrier germanoaméricain; deuxièmement, l'ensemble des modèles identitaires véhiculés par ces périodiques; troisièmement, les formes possibles de communication interculturelle; et, finalement, la relation entre adaptation culturelle et autonomie.

Publié en 1993 par Walter Killy dans Literaturlexikon², le seul article général relativement récent qui propose un point de vue d'ensemble sur les relations littéraires entre l'Amérique du Nord et l'Allemagne n'a pas tenu compte d'une importante source

1. Cet article s'inscrit dans un projet DFG conjoint avec Hans-Jürgen Lüsebrink (Universität des Saarlandes), sous le titre de «Cultural Transfer and Process of Autonomy. Comparative and intercultural studies of German-American and Franco-Canadian eighteenth and nineteenth century popular calendars and almanacs». Traduit de l'anglais par Stéphanie Massé et Yves Bourassa.

2. Walter Schmitz, "Nordamerikanisch-deutsche Literaturbeziehungen », dans Walther Killy (sous la dir. de), Literaturlexikon, vol. 14, Gütersloh, Munich, Bertelsmann Lexikon Verlag, 1993, p. 162-170. 
textuelle pourtant inscrite au cœur du discours social le plus quotidien et étroitement liée aux questions d'identité et de différences culturelles: celle des calendriers populaires allemands publiés en Pennsylvanie (à partir de 1731) et ailleurs en Amérique du Nord. Le fait d'ignorer ces périodiques a favorisé le développement de l'idée suivant laquelle les «immigrants", tout comme les Américains d'origine allemande dont l'influence s'exerça après 1848 , n'ont pas participé au "discours culturel américain», en plus de mener à la conclusion que les transferts culturels furent d'abord, sinon uniquement, la conséquence de voyages entrepris par des Européens cultivés. De telles conclusions sont contestables à la lumière du Meyer's Groschen-Bibliothek der Deutschen Classiker, une collection de petits livres qui s'adressaient aux immigrants. Publiés à Hildburghausen et à New York, ces ouvrages étaient destinés à la consommation de masse. Il faudrait également mentionner le programme littéraire de l'Amerikanische Tractat-Gesellschaft, qui compte plus de 160 titres, les nombreux périodiques allemands et au moins trente-neuf séries de calendriers allemands publiés dans l'est de l'Amérique du Nord. Le volume 15 de Grundriß zur Geschichte der Deutschen Dichtung ${ }^{3}$ de Karl Goedeke, édité par Herbert Jacob en 1966, présente pour sa part un survol de la littérature germano-américaine en 142 pages.

En Amérique du Nord, la librairie allemande est un commerce concentré à l'origine en Pennsylvanie, c'est-à-dire dans un état qui fut d'abord une colonie britannique. En 1775, le nombre d'immigrants germanophones y dépassait de beaucoup les 100000 habitants, si bien qu'une culture non anglophone florissait alors à

3. La série Meyer's Groschen-Bibliothek der Deutschen Classiker était composée d'environ cent petits volumes; les mentions de l'éditeur demeuraient toujours les mêmes: «Hildburghausen: Druck vom Bibliographischen Institut. New York: Hermann J. Meyer». Si l'on en juge par la couverture, cette série a été envoyée à «tous les libraires d'importance en Allemagne et ailleurs». À lire les réclames de l'éditeur, l'Amerikanische Tractat-Gesellschaft, basé à New York, offrait un grand nombre de "Erbauungs-Bücher von ausgezeichneten theologischen Schiftstellern, sowie 140 evangelische Tractate und 20 Kinder-Tractate» ( livres d'édification signés par d'éminents théologiens, aussi bien que 140 traités évangélistes et 20 ouvrages pour la jeunesse »). Pour les périodiques et les livres germano-américains, voir Karl Goedeke, 1, Bd. XV, Berlin, Herbert Jacob Akademie Verlag, 1966, p. 518-561; l'immigration dans la fiction contemporaine est étudiée par Juliane Mikoletzky dans Die deutsche Amerika-Auswanderung des 19. Jahrhunderts in der zeitgenössischen fiktionalen Literatur, Tübingen, Max Niemeyer, 1988. 
Germantown, Lancaster et Philadelphie ${ }^{4}$. Le premier calendrier germano-américain, qui figure dans l'incontournable bibliographie de Karl John Arndt et May Evelyn Olson, le DeutschAmerikanische Zeitungen und Zeitschriften 1732-1955, a été publié en 1731 sous le titre de Der Teutsche Pilgrim, Mitbringende einen Sitten-Calender. La plupart des publications annuelles complètes ont vu le jour dans les décennies qui ont suivi l'année 1776. La majeure partie d'entre elles - trente-quatre séries - ont paru en Pennsylvanie, et cinq autres en Ohio et au Maryland. Environ le quart de ces périodiques ont eu cours pendant une période de temps considérable: pour preuve, le Hoch-Deutsche Americanische Calender (Germantown et Philadelphie), qui fut «certainement le plus important ${ }^{5}$ », parut de 1739 à 1833.

En plus des rubriques destinées au calendrier, ces publications comportaient également une section littéraire où l'on présentait habituellement quelques titres parus en Allemagne, aussi bien que des contributions originales de producteurs de calendriers ou d'auteurs de langue allemande résidant en Amérique du Nord. Les orientations intellectuelles se voulaient éducatives et émancipatrices, portées par un esprit égalitaire et, bien souvent aussi, abolitionniste ${ }^{6}$.

Dès leur origine, ces calendriers s'intéressaient résolument à des sujets américains. Les textes littéraires en particulier mettent en évidence le processus d'autonomisation culturelle. Comme il ne s'agit en aucun cas d'un processus transitionnel simple et univoque, il semble quelquefois difficile de déterminer si ces publications appartiennent à la culture allemande ou nord-américaine ${ }^{7}$.

4. Déjà 1800 imprimeurs et libraires, tel Jacob Hütter à Lancaster, offraient un choix de « 8000 ouvrages» parmi les "plus récents et les meilleurs livres allemands »; voir Karl Goedeke, Grundriß, ouvr. cité, p. 519. Le lectorat francophile à Philadelphie bénéficiait des services de "Boinod et Gaillard", qui publiaient depuis 1784 un Catalogue des livres renfermant un nombre exceptionnel de titres d'auteurs français; voir Madeleine B. Stern, «Ursprung und Entwicklung des Antiquariatsbuchhandels in Philadelphia», dans Aus dem Antiquariat, 11, 1984, A408.

5. Karl Goedeke, Grundriß, ouvr. cité, p. 541: "definitely the most important" (nous traduisons).

6. Sur ce point, voir York-Gothart Mix, «Lektüre für Gebildete und Ungebildete. Einleitende Bemerkungen zu H. C. Boies “Musenalmanach”, J.P. Hebels "Rheinländischem Hausfreund" und anderen literarischen Begleitern durch das Jahr», dans York-Gothart Mix (sous la dir. de), Almanach- und Taschenbuchkultur des 18. und 19. Jahrhunderts, Wiesbaden, Harrassowitz, 1996, p. 16 et suiv.

7. Voir Karl Goedeke, Grundriß, ouvr. cité, p. 519. 
La question que pose Karl Kurt Klein dans Literaturgeschichte des Deutschtums im Ausland (1939), et qui cherche à mesurer jusqu'à quel point la culture germano-américaine prend appui sur un Volkskörper germanique, reste vaine étant donné ses présupposés racistes et organicistes ${ }^{8}$. Se concentrer sur la qualité esthétique des textes germano-américains, comme le suggère Jacob ${ }^{9}$, semble également insatisfaisant puisque la dimension sociale de la valeur symbolique du transfert culturel ${ }^{10}$ se trouve alors marginalisée.

Aussi bien le nom que le programme que se donne un périodique comme le Hinckend-und Stolpernd-doch eilfertig-fliegend-und laufend Americanische Reichs-Bott ${ }^{11}$ illustre les tensions et les contradictions entre transfert culturel et autonomie. L'entreprise procédait de l'exemple offert par un calendrier publié depuis au moins 1698 dans la ville allemande d'Offenbach et intitulé Der Hinckend-und Stolpernd-doch eilfertig fliegend-und lauffende Bott. Das ist: Neu-Verbesserter Reichs-Staats-Geschichts-Kriegs-Siegs-und Friedens-Calender. Semblable au Cincinnatier hinkende Bote, qui paraîtra presque un siècle plus tard, il se voulait à l'origine un vade-mecum rédigé en allemand "pour les États-Unis d'Amérique du Nord ${ }^{12} »$. Mais ce qui apparaissait comme un périodique absolument traditionnel va présenter des différences significatives en dépit des similarités de mise en pages, de concepts et de contenus.

Ce n'est plus, désormais, aux élites dirigeantes ou à leurs sujets que le messager claudiquant figurant sur la page de titre apporte

8. Voir Karl Kurt Klein, Literaturgeschichte des Deutschtums im Ausland. Schrifttum und Geistesleben der deutschen Volksgruppen im Ausland vom Mittelalter bis zur Gegenwart, Leipzig, Bibliographisches Institut, 1939, p. 87.

9. Voir Karl Goedeke, Grundriß, ouvr. cité, p. 518.

10. Voir Georg Bollenbeck, Bildung und Kultur. Glanz und Elend eines deutschen Deutungsmusters, Frankfurt/Main, Leipzig, Insel Verlag, 1994, p. 193; Sabine A. Döring, "Vom "nation-building" zum Identifikationsfeld. Zur Integrationsfunktion nationaler Mythen in der Literatur», dans Horst Turk, Brigitte Schultze et Roberto Simanowski (sous la dir. de), Kulturelle Grenzziehungen im Spiegel der Literaturen. Nationalismus, Regionalismus. Fundamentalismus, Göttingen, Wallstein, 1998, p. 75 et suiv.; Joseph Jurt, «Das Konzept des literarischen Feldes und die Internationalisierung der Literatur», dans Horst Turk, Brigitte Schultze et Roberto Simanowski (sous la dir. de), Kulturelle Grenzziehungen, ouvr. cité, p. 101 et suiv.

11. Voir ici Milton Drake (édition préparée par), Almanacs of the United States. Part II, New York, The Scarecrow Press, 1962, p. 963; pour les calendriers germano-américains et les almanachs, voir aussi Goedeke, Grundri $\beta$, ouvr. cité, p. 540-544.

12. Cincinnatier hinkende Bote, 1865 , page-titre. 
les dernières nouvelles, mais à des colons recherchant rendements et indépendance sous les ailes protectrices de l'aigle américain, entouré d'étoiles; quant à la page frontispice du Cincinnatier hinkende Bote, elle montrait un grand lopin de terre non cultivée et une cabane en bois rond nouvellement construite. L'iconographie de la page de titre illustre clairement un remodelage en cours de l'identité socioculturelle. La vie du lecteur auquel on entendait s'adresser s'inscrivait dorénavant dans un contexte régional aussi bien que global, où des symboles appartenant à toute une communauté évoquaient déjà une histoire partagée.

La complexité de l'histoire se trouvait en partie évacuée en raison de la sélection et de la combinaison de faits singuliers et d'événements présidant à la formation identitaire, ce qui ouvrait ainsi de nouvelles voies à l'expérience historique. La personnification du calendrier devenait l'objet d'une auto-représentation conçue de manière à corroborer la vérité des faits rapportés, tout en signifiant l'intention de transformer en un schème familier tout ce qui pouvait s'apparenter à l'étrange ou à l'étranger. Les textes prolongeaient toujours un schème qui popularisait une nouvelle conscience de l'histoire et de la culture, et ce, en dépit de cette "tendance à persister» déjà étudiée par Rudolf Schenda ${ }^{13}$. La réalité n'était pas interprétée en fonction de simulacres d'ordre esthétique, ni même réflétée comme réalité, mais construite d'une façon spécifique au média ${ }^{14}$.

En corrélation avec sa propre histoire et en fonction de ce qui se conçoit comme la culture singulière d'une collectivité, la légitimation de l'identité à l'intérieur des dynamiques discursives propres à une sphère sociale et politique distincte reçut enfin ses lettres de noblesse. Pour le monde européen, tout cela évoquait divers concepts relatifs aux devoirs que se doivent d'accomplir les citoyens et à la place que chacun occupe dans la société et dans l'histoire, ce qui ne manqua pas d'avoir bientôt un impact sur le Vieux Continent et dans l'ancienne patrie. C'est ce que montre, par exemple, le fait qu'on regardât comme une référence décisive un «Moralischer Artikel» publié dans le Pennsylvanischer Haushaltskalender de 1770: celui-ci est réimprimé dans le Hannoveranisches Magazin en 1771 et, à nouveau, à la satisfaction de tous,

13. Rudolph Schenda, Volk ohne Buch. Studien zur Sozialgeschichte der populären Lesestoffe 1770-1910, Frankfurt/Main, Klostermann, 1970, p. 325.

14. Voir Niklas Luhmann, Die Realität der Massenmedien, Opladen, Westdeutscher Verlag, 1966, p. 16 et suiv. 
dans le Siebenbürgischer Almanach de Hermannstadt en $1781^{15}$. En revanche, Heinrich Heine devait rejeter cette conception positive de l'Amérique qu'avait même envisagée Johann Wolfgang Goethe dans ses Zahmen Xenien et son Wilhelm Meisters Wanderjahre. À l'image goethéenne d'une Amérique englobant un «champ illimité de questions générales sur l'éthique et la moralité ${ }^{16}$ » et une "volonté inconditionnelle de travailler ${ }^{17}{ }^{»}$, Heine oppose, dans un traité polémique comme Ludwig Börne, les termes aussi frappants que péjoratifs d' "empire sans merci», d' «esclavage», de "brutalité», d' "hypocrisie», d' "égoïsme» et d' «injustice», avant de conclure que «le profit matériel représente leur vraie religion et l'argent leur Dieu, leur seul Dieu omnipotent ${ }^{18}{ }^{»}$.

Dans l'ombre du classicisme de Weimar s'est propagée une « esthétique ennoblissante de l'existence bourgeoise, privée de forme et prosaïque ${ }^{19}$ ", en même temps que l'éducation devenait un idéal que l'on célébrait comme une "caractéristique nationale ${ }^{20} »$. Dans les textes consacrés à l'histoire littéraire, le but ultime de la culture était résolument lié à sa dimension nationale. En Amérique du Nord, la culture allemande s'est cependant développée dans des conditions bien différentes. Les immigrants percevaient l'absence d'État comme une garantie de la "liberté d'expression et de pensée ${ }^{21} »$. Par conséquent, il n’y a jamais eu de

15. Voir Wilhem Haefs et York-Gothart Mix, «Der Musenhort in der Provinz. Literarische Almanache in den Kronländern der österreichischen Monarchie im ausgehenden 18. und beginnenden 19. Jahrhundert », Archiv für Geschichte des Buchwesens (AGB), 27, 1986, p. 184.

16. Wilhelm Meisters Wanderjahre, dans Johann Wolfgang Goethe, Sämtliche Werke. I. Abt., Bd. 10, édition préparée par G. Neumann et H.-G. Dewitz, Frankfurt/Main, Deutscher Klassiker Verlag, 1989, p. 343.

17. Johann Wolfgang Goethe, Wilhelm Meisters Wanderjahre, ouvr. cité.

18. Ludwig Börne: Eine Denkschrift, dans Henrich Heine, Historisch-kritische Gesamtausgabe der Werke, Düsseldorfer Ausgabe, édition préparée par Helmut Koopmann, Hambourg, Hoffmann und Campe, 1978, tome XI, p. 37 et suiv.

19. Dieter Borchmeyer, Weimarer Klassik. Portrait einer Epoche, Weinheim, Belz, 1994, p. 295.

20. Georg Bollenbeck, Bildung und Kultur, ouvr. cité, p. 160.

21. "Time is money", "John Bull, der Enthusiast», dans August Heinrich Hoffmann von Fallersleben, Gedichte und Lieder, édition préparée par Hermann Wendebourg et Anneliese Gerbert, Hamoburg, Hoffmann und Campe, 1974, p. 302; Alexander Ritter, «Grenzübertritt und Schattentausch. Der österreichische Priester Carl Postl und seine vage staatsbürgerliche Identität als amerikanischer Autor Charles Sealsfield. Eine Dokumentation ", dans Freiburger Universitätsblätter, 143, 1999, p. 39 et suiv. 
classe bourgeoise cultivée faisant corps avec l'État, pas plus qu'il n'y a eu de crise culturelle marquante en réaction à l'idéalisation, dépourvue de sens critique, de l'utopie éducative qui apparut après $1871^{22}$. Au contraire, la réception d'une culture allemande et autrichienne d'élite dans les communautés allemandes ${ }^{23}$ était centrée sur la littérature populaire allemande ${ }^{24}$ et inscrite dans les structures communicationnelles d'un «réseau civilisant» qui a été tourné en dérision en Allemagne pendant plusieurs décennies, attestant la «fatale antithèse entre Kultur et culture ${ }^{25}$ ».

Lors de son voyage en Amérique en 1831, Alexis de Tocqueville prétendait que les Nord-Américains ne possédaient «encore, à strictement parler, aucune littérature ${ }^{26} »$. Cette prétention a été formulée à la lumière de la distribution sur une grande échelle de calendriers et de périodiques populaires, de la réception des ouvrages européens canoniques et de l'émergence tardive d'une littérature américaine originale. À la différence de l'Europe, l'identité nationale, aux États-Unis, n'est pas le fruit d'une culture intégrante soutenue par l'État. D'un point de vue politique et social, l'«Américain» était déjà considéré, dès le XVIII siècle, comme «un nouvel être»; cependant, les traditions culturelles de la patrie

22. Voir York-Gothart Mix, Die Schulen der Nation. Bildungskritik in der Literatur der frühen Moderne, Stuttgart, Weimar, B. Metzler, 1995, p. 26 et suiv. et p. 185 et suiv.

23. Voir Kathleen Neils Conzen, "Patterns of German-American History», dans Randall M. Miller (sous la dir. de), Germans in America. Retrospect and Prospect. Tricentennial Lectures Delivered at the German Society of Pennsylvania, Philadelphia, The German Society of Pennsylvania, 1984, p. 27 et suiv.

24. Voir Reinhard R. Doerris, "Immigrant Culture and Religion. Church and Faith Among German Americans», dans Randall M. Miller (sous la dir. de), Germans in America, ouvr. cité, p. 81 et suiv.; Don Yoder, "The Palatine Connection. The Pennsylvania German Culture and Its European Roots », dans Randall M. Miller (sous la dir. de), Germans in America, ouvr. cité, p. 94 et suiv.; Curtis C. Bentzel, "Calligraphy and Linguistics. A Different Look at the F\&M Fraktur", dans Fraktur. A Selective Guide to the Franklin and Marshall Fraktur Collection, Lancaster, Franklin and Marshall College, 1987, p. 9 et suiv.; Paul Conner et Jill Roberts (sous la dir. de), Pennsylvania German Fraktur and Printed Broadsides. A Guide to the Collections in the Library of Congress, Washington, Library of Congress, 1988.

25. "Auf die Frage: Was ist deutsch", dans Theodor W. Adorno, Gesammelte Schriften, Bd. 10, 2, édition préparée par Rolf Tiedemann, Frankfurt/Main, Suhrkamp, 1977, p. 695 et p. 697.

26. Alexis de Tocqueville, "Über die Demokratie in Amerika », dans Heinz Förster (sous la dir. de), Was ist ein Amerikaner? Zeugnisse aus dem Zeitalter der amerikanischen Revolution, Leipzig et Weimar, Gustav Kiepenheuer, 1987, p. 342. 
allemande continuaient à exercer une influence qui façonnait l'identité. À l'aube de la Révolution française, Hector St. John de Crèvecœur écrivait, à propos des immigrants allemands en Pennsylvanie:

Leur étonnement était grand à leur arrivée d'Allemagne - cela leur semble être un rêve; le contraste doit être en effet saisissant; ils observent partout leurs compatriotes florissants qui prospèrent; ils traversent de vastes régions où l'on n'entend pas un mot d'anglais; et, dans les noms et le langage des gens, ils retrouvent l'Allemagne. Ils ont rendu de grands services à ce continent, et à la Pennsylvanie en particulier; elle leur doit une partie de sa prospérité: à leurs connaissances mécaniques, à leur patience aussi, elle doit les meilleurs moulins de toute l'Amérique, les meilleurs attelages de chevaux et beaucoup d'autres avantages. Le souvenir de leur pauvreté et de leur esclavage passés ne les quitte jamais tout au long de leur vie ${ }^{27}$.

Mieux qu'aucun autre média, le calendrier populaire permet de représenter, dans le vécu quotidien, le paradoxe apparent qui survient entre le traditionalisme culturel et le sens de l'identité régionale, d'une part, la modernité économique et politique, d'autre part. Des calendriers tels que le Hinkende Bote ou le Haushaltungs-Kalender ${ }^{28}$ ont prouvé leur efficacité comme médias favorisant l'intégration ethno-culturelle en Europe. Alors que «peu

27. "Their astonishment at their first arrival from Germany is very great - it is to them a dream; the contrast must be very powerful indeed; they observe their countrymen flourishing in every place; they travel through whole counties where not a word of English is spoken; and in the names and the language of the people, they retrace Germany. They have been an useful acquisition to this continent, and to Pennsylvania in particular; to them it owes some share of its prosperity: to their mechanical knowledge and patience it owes the finest mills in all America, the best teams of horses, and many other advantages. The recollection of their former poverty and slavery never quits them as long as they live.» J. Hector St. John de Crèvecœur, Letters from an American Farmer and Sketches of EighteenthCentury America [1782], édition préparée par Albert E. Stone, New York, Penguin Books, 1981, p. 78. Pour la traduction française, voir Pierre Monette, «St. John de Crèvecœur et les Lettres d'un fermier américain. Traductions des Lettres d'un fermier américain (1782) et de "Description d'une tempête de neige au Canada" (ca. 1780), précédées d'une présentation et de notes explicatives suivies d'une étude sur les échos du séjour de St. John de Crèvecœur en Nouvelle-France dans les Lettres d'un fermier américain", Thèse de doctorat, Montréal, Université du Québec à Montréal, 2003, p. 229-230.

28. Voir Inga Wiedemann, "Der Hinkende Bote" und seine Vettern. Familien-, Haus- und Volkskalender von 1757 bis 1929. Katalog der Kalendersammlung des Museums für Deutsche Volkskunde, Berlin, Museum für Deutsche Volkskunde, 1984, p. 51 et suiv. 
d'immigrants allemands savaient la langue anglaise à leur arrivée aux États-Unis ${ }^{29}$ ", le calendrier continuait d'être un guide important dans le Nouveau Monde. Comme les romans à l'usage des immigrants du XIX ${ }^{e}$ siècle, beaucoup de calendriers populaires enseignaient à leurs lecteurs qu'une adaptation culturelle forcée ne représentait pas un objectif important:

seuls les personnages nourrissant des intentions criminelles s'adaptent rapidement et volontairement au mode de vie américain; [...] d'autre part, les habitudes et les objets venus d'Allemagne et que l'on a conservés servent généralement à décrire favorablement les personnages ${ }^{30}$.

Quand les révolutions de 1848 échouèrent, les attitudes antiaméricaines, affichées par certains auteurs de la jeune école allemande comme Heinrich Heine, se sont cristallisées en une idée de «supériorité culturelle» et, en bout de ligne, ce sentiment a alimenté une "peur des éléments étrangers chez beaucoup d'Américains $^{31} »$.

Comme dans les romans de l'immigration, les calendriers présentaient une image de soi positive, elle-même fondée sur les qualités allemandes présumées, qu'il s'agisse de l'intégrité, de l'industrie, de l'honnêteté et du respect de la loi. Pour beaucoup d'immigrants, insister sur des normes et des valeurs distinctives renforçait la conscience d'une différence ethno-culturelle qui, à l'occasion, sera dirigée à dessein contre la mère-patrie, considérée comme arriérée.

Au sein d'un contexte politique différent, une identité culturelle divergente s'est développée dans le Nouveau Monde. Favorisant un "esprit de $\operatorname{clan}^{32}$ ", elle correspondait à une attitude d'autoprotection fondamentale, laquelle engendra l'idée suivant laquelle les Germano-Américains incarnaient, pour la première fois dans l'histoire, ce qui était réellement allemand. Friedrich Gerstäcker, un auteur de récits de voyage qui a quitté les ÉtatsUnis pour retourner chez lui en 1843, écrit dans son livre In Amerika, publié en 1879:

Ce que nous n'avons jamais été en Allemagne, nous le sommes soudainement devenus ici: de vrais Allemands - sans tout ce

29. Juliane Mikoletzky, Die deutsche Amerika-Auswanderung, ouvr. cité, p. 253.

30. Juliane Mikoletsky, Die deutsche Amerika-Auswanderung, ouvr. cité, p. 272.

31. Juliane Mikoletsky, Die deutsche Amerika-Auswanderung, ouvr. cité, p. 277.

32. Juliane Mikoletsky, Die deutsche Amerika-Auswanderung, ouvr. cité, p. 300. 
misérable préjugé de la terre natale qui ne peut survivre au voyage. Nous sommes devenus non seulement Allemands, mais aussi des gens avec une conscience de l'égalité pour tous ceux qui accomplissent quelque chose; simultanément se faisait jour une chose que, de retour à la maison, plusieurs d'entre nous n'avaient jamais connu que de nom: le respect de $\operatorname{soi}^{33}$.

\section{Questions pour une recherche future}

Le contexte que l'on vient d'esquisser définit quatre objets qui seront de la plus haute importance pour toute recherche future. Il s'agit d'étudier d'abord les caractéristiques spécifiques du média et les différences génériques qui s'expriment dans le calendrier germanoaméricain; deuxièmement, l'ensemble des modèles identitaires véhiculés par ces périodiques; troisièmement, les formes possibles de communication interculturelle; et, finalement, la relation entre adaptation culturelle et autonomie.

Le premier faisceau de problèmes inclut l'analyse de la sociologie du lectorat et de l'histoire du commerce de la librairie qui a rendu possible une forme écrite de communication à vaste échelle dans les régions germanophones des États-Unis. L'accent devrait être mis sur les différences avec les schèmes des almanachs populaires européens: le profil des lecteurs implicites et explicites, les genres textuels, l'iconographie calendaire, la relation entre texte et illustration, les intentions de l'éditeur, l'attention portée à la vie quotidienne en Amérique, l'insistance sur des textes à prétention morale et didactique, la préférence accordée aux nouveaux genres, comme les dialogues entre immigrants, ainsi que la construction d'une nouvelle lecture de l'histoire.

Au regard de la constitution d'une histoire américaine qui leur est propre, on devra aussi examiner, deuxième faisceau de problèmes, les relations entre les histoires et l'histoire. À cet égard, les écrits des immigrants se rapportant à la vie quotidienne, où se réflétait un sujet à la fois individuel et paradigmatique, devront se lire aussi bien comme parties constitutives d'un ensemble de modèles identitaires collectifs que comme formes symboliques traditionnelles de codification. Fatalement, l'âge des calendriers américains correspond à une période circonscrite à quelques générations; les images et les histoires dérivées d'un temps préhis-

33. Friedrich Gerstäcker, In Amerika. Amerikanisches Lebensbild aus neuerer Zeit. Im Anschluss an "Nach Amerika", Bd. I, Iéna, Fromann, 1879, vol. I, p. 233. 
torique et mythique ${ }^{34}$, largement diffusées dans le contexte de la construction d'une nation allemande, ne jouent aucun rôle dans les calendriers germano-américains.

Le sentiment de vivre un renouveau crucial et mémorable a stimulé une "unification ${ }^{35}$ » qui était à peu près inconnue en Europe: par-delà les conflits confessionnels, historiques et territoriaux, monarchiques ou relatifs à l'administration de l'État, les calendriers fonctionnaient comme un perpetuum mobile agissant sur les modèles d'identification historiques et ethno-culturels. Dans le processus d'analyse des contenus, il importe de s'attarder sur des questions touchant la formation de références et de thèmes historiques puissamment évocateurs, la relation entre événements historiques et fiction, la place centrale accordée aux catégories historico-téléologiques, les stéréotypes présidant à une interprétation globale de l'univers, le caractère propre à des textes vraisemblablement destinés à d'abord interpeller le lectorat, tout comme le brouillage des frontières entre langue orale et écrite.

Troisièmement, les modèles d'identification ainsi codifiés permettront d'apporter un nouvel éclairage tant sur les références intraculturelles à la tradition que sur les relations interculturelles. Cette concurrence latente entre divers modèles se manifeste sur le plan de l'orthographe, de la syntaxe, du vocabulaire, des anglicismes et des idiomatismes germaniques, aussi bien que dans les exemples de migration textuelle, de traductions, de renseignements administratifs et de publicités faisant la réclame de livres, elles-mêmes typiques des calendriers. Les calendriers germano-américains ne représentent pas seulement les marges d'une communication quotidienne écrite: ils illustrent encore la manière dont des textes typiques d'une époque ou hautement estimés en vinrent à former une partie intégrante de la culture populaire.

Le quatrième faisceau de problèmes concerne la relation entre intégration culturelle et autonomie. Même si, au début, les «Hessois, Francfortois, Westfalois, Hanovriens et Oldenbourgeois» qui

34. Voir Dieter Langewiesche, «Kulturelle Nationenbildung im Deutschland des 19. Jahrhunderts», dans Manfred Hettling et Paul Nolte (sous la dir. de), Nation und Gesellschaft in Deutschland. Historische Essays, Munich, C.H. Beck, 1996, p. 58; Hans-Martin Blitz, Aus Liebe zum Vaterland. Die deutsche Nation im 18. Jahrhundert, Hambourg, Hamburger Edition, 2000, p. 361 et suiv.

35. Thomas Schmidt, Kalender und Gedächtnis. Erinnern im Rhythmus der Zeit, Göttingen, Wandenhoeck \& Ruprecht, 2000, p. 35. 
ont suivi les «Rhénans, Badois et Würtembourgeois ${ }^{36}$ » ne formaient pas un groupe possédant une identité propre au-delà de celle que leur conférait leur statut d'immigrants, ils étaient considérés aux États-Unis « comme de simples Allemands ${ }^{37}$ ». Toutefois, cette tendance à simplifier à l'excès le concept en fonction duquel se trouvent définis le soi et l'autre était réciproque, et de même que l'on retrouvait chez les Germano-Américains une représentation de soi différenciée, de même y avait-il à l'opposé l'image éclatée que donne de lui-même le Nord-Américain qui n'est pas d'ascendance allemande. L'autonomie culturelle était issue de la conscience de la différence qui, suivant la maxime de Carl Schurz, «Ubi libertas, ibi patria» ("Là où se trouve la liberté se trouve aussi la patrie»), impliquait aussi une très nette mise à distance avec la mère-patrie. La «définition de l'ethnicité était essentiellement culturelle $^{38} »$. Elle n'était pas le fruit d'ambitions impérialistes orientées vers la conquête du pouvoir, mais représentait clairement une "protestation contre les conditions politiques prévalant dans la patrie allemande, une sorte de vote qu'en s'expatriant, leurs pieds, pour ainsi dire, exprimaient ${ }^{39}{ }^{\prime}$.

$\mathrm{Au}$ regard des discussions qui avaient cours en Allemagne sur l'éducation, il reste, par conséquent, à voir si l'interprétation allemande de l'identité culturelle qu'a proposée Georg Bollenbeck et qui exerça une si forte influence sur le XIX ${ }^{e}$ siècle ne serait pas aussi apodictique et libre de contradictions que peut l'être une synthèse amalgamant l'éducation à une modernisation politiquement réactionnaire; plus encore, si l'identité culturelle peut être encore définie comme «la conscience d'une tâche exceptionnelle à accomplir ${ }^{40} »$. Dans un contexte où l'on accorda la préférence à un concept universel d'éducation plutôt qu'à un programme de formation centré sur les besoins du marché, les premières images et les premiers symboles culturels stables alors en émergence aux États-Unis étaient d'une nature sociale et politique entièrement différente:

36. Kathleen Neils Conzen, "Patterns of German-American History», art. cité, p. 20.

37. Kathleen Neils Conzen, «Patterns of German-American History», art. cité, p. 25.

38. Kathleen Neils Conzen, «Patterns of German-American History», art. cité, p. 29.

39. Hans Ottomeyer (sous la dir. de), Biedermeiers Glück und Ende ... die gestörte Idylle 1815-1848, Munich, Hugendubel, 1988, p. 551.

40. Georg Bollenbeck, Bildung und Kultur, ouvr. cité, p. 162. 
Les Germano-Américains défendaient constamment leur différence en soutenant l'argument qu'ils désiraient préserver certaines valeurs de façon à participer à une culture nouvelle et véritablement américaine, et ils n'ont réussi que trop bien. Dès le début du $\mathrm{XX}^{\mathrm{e}}$ siècle, les écoles et les universités américaines fournissaient une éducation supérieure différant à peine de celle dispensée dans les institutions allemandes ${ }^{41}$.

Les «caractéristiques nationales» étaient déterminées par une conception de l'éducation qui incluait une double perspective sur le soi et l'autre; le critère de Bollenbeck d'une «fonction politique déficiente ${ }^{42}$ », pourtant, n'est en aucun cas une conséquence inévitable lorsqu'il s'agit de comprendre la culture dans sa dimension collective. L'idée d'une formation centrée sur l'individu est constitutive des calendriers germano-américains, eux-mêmes fondés sur une conception de la réalisation harmonieuse de soi, laquelle est ultimement modelée par un idéalisme qui conçoit l'éducation comme conquête d'une autonomie. Sur le plan de la recherche littéraire, plusieurs exemples ilustrent ce processus, qu'il s'agisse d'un texte en prose comme «Der Deutsche Patriot» (1801) et du genre du traité politique à caractère programmatique ${ }^{43}$ ou encore de dialogues d'immigrants et de textes calendaires faisant allusion à des événements historiques.

\section{Corpus des Calendriers et Almanachs germano-américains cités}

Americanischer Stadt und Land Calender Auf das 1830ste Jahr Christi, Welches ein Gemeines Jahr ist von 365 Tagen, Philadelphie, Gedruckt und zu haben bey Conrad Zentler, in der Zweyten Strasse, unterhalb der Rehs-Strasse, non paginé.

Americanischer Stadt und Land Calender Auf das 1840ste Jahr Christi, Welches ein Schalt=Jahr ist von 366 Tagen, Philadelphie, Gedruckt und zu haben bey Conrad Zentler, in der Zweyten Strasse, unterhalb der Rehs-Strasse, non paginé.

Der Americanische Stadt- und Land-Calender auf das Jahr 1852, Philadelphie, Herausgegeben von Ch. G. Sauer, non paginé.

41. Kathleen Neils Conzen, «Patterns of German-American History», art. cité, p. 33.

42. Georg Bollenbeck, Bildung und Kultur, ouvr. cité, p. 162.

43. Voir Der Zustand der Deutschen in Pennsylvanien im Jahr 1825, dans Der Hoch-Deutsche Americanische Calender, 1786. 
Der Cincinnatier hinkende Bote. Ein Kalender für die Vereinigten Staaten von Nordamerika, auf das Jahr unsers Herrn 1865, welches ein gemeines Jahr von 365 Tagen ist, Cincinnati, Verlag von Gebrüder Benzinger, Cincinnati, im Katholischen Institut, New York, $\mathrm{n}^{\circ} 9$ Dey Str.

Der gemeinnützige Americanische Calender Auf das Jahr Christi 1803, Reading, gedruckt und zu haben bey Schneider und Ritter, non paginé.

Der gemeinnützige Americanische Calender Auf das Jahr Christi 1801, Reading, gedruckt und zu haben bey Jacob Schneider und Comp, non paginé.

Der Hoch-Deutsch Americanische Calender, Auf das Jahr Nach der Gnadenreichen Geburt unsers HErrn und Heylandes JEsu Christi 1748, Germantown, Gedruckt und zu finden bey Christoph Saur, non paginé.

Der Neue Hoch Deutsche Americanische Calender, Auf das Jahr Christi 1798, Welches ein Gemein Jahr von 365 Tagen ist, Baltimore, gedruckt und zu finden bey Samuel Saur, non paginé.

Der Zustand der Deutschen in Pennsylvanien im Jahr 1825. Wenn sie Wollen, dans Der Hoch-Deutsche Americanische Calender, Auf das Jahr 1786, Germantaun, gedruckt und zu finden bey Leibert und Billmeyer, non paginé.

\section{Autres références non citées}

Brauer, Adalbert, "Friedrich Kapp und die Geschichte des deutschen Buchhandels », Buchhandelsgeschichte 4, Frankfurt/ Main, Historische Kommission des Börsenvereins, 1984, B. 150156.

Esser, Hartmut, Aspekte der Wanderungssoziologie. Assimilation und Integration von Wanderern, ethnischen Gruppen und Minderheiten. Eine handlungstheoretische Analyse, Darmstadt, Neuwied, Luchterhand, 1980.

Hobsbawm, Eric, "Das Erfinden von Traditionen ", dans Christoph Conrad et Martina Kessel (sous la dir. de), Kultur \& Geschichte. Neue Einblicke in eine alte Beziehung, Stuttgart, Reclam, 1998, p. 97-118.

Jeisman, Karl-Ernst, "Zur Bedeutung der "Bildung” im 19. Jahrhundert", dans Handbuch der deutschen Bildungsgeschichte, 
vol. III. 1800-1870, dans Karl-Ernst Jeisman et Peter Lundgreen (édition préparée par), Von der Neuordnung Deutschlands bis zur Gründung des Deutschen Reiches, Munich, C.H. Beck, 1987, p. 121.

Koselleck, Reinhart, "Geschichte», dans Otto Brunner, Werner Conze et Reinhart Koselleck (sous la dir. de), Geschichtliche Grundbegriffe. Historisches Lexikon zur politisch-sozialen Sprache in Deutschland, vol. II, Stuttgart, Klett-Cotta, 3. Aufl., 1992, p. 593717.

Masel, Katharina, Kalender und Volksaufklärung in Bayern. Zur Entwicklung des Kalenderwesens 1750 bis 1830, St. Ottilien, Eos, 1997. 